\title{
Salvage treatment for children with relapsed/refractory germ cell tumors: The Associazione Italiana Ematologia Oncologia Pediatrica (AIEOP) experience
}

\author{
Maria Debora De Pasquale ${ }^{1}$ (i) | Paolo D'Angelo² | Alessandro Crocoli ${ }^{3}$ (iD | \\ Renata Boldrini $^{4}$ | Massimo Conte ${ }^{5}$ | Gianni Bisogno ${ }^{6}$ (D) | Filippo Spreafico ${ }^{7}$ (iD | \\ Alessandro Inserra $^{3}$ (D) | Davide Biasoni ${ }^{7}$ | Patrizia Dall'Igna ${ }^{8}$ (D) \\ Fortunato Siracusa $^{9}$ | Evelina Miele ${ }^{1}$ (i) | Monica Terenziani ${ }^{7}$ (iD)
}

${ }^{1}$ Hematology/Oncology Department, Ospedale Pediatrico Bambino Gesù, IRCCS, Roma, Italy

${ }^{2}$ Pediatric Hematology/Oncology Unit, A.R.N.A.S. Civico Di Cristina e Benfratelli, Palermo, Italy

${ }^{3}$ Surgery Department, Ospedale Pediatrico Bambino Gesù, IRCCS, Roma, Italy

${ }^{4}$ Pathology Unit, Ospedale Pediatrico Bambino Gesù, IRCCS, Roma, Italy

${ }^{5}$ Oncology Unit, IRCCS Istituto G. Gaslini, Genova, Italy

${ }^{6}$ Pediatric Oncology, Women's and Children's Health Department, University of Padua, Padua, Italy

${ }^{7}$ Pediatric Oncology Unit, Fondazione IRCCS Istituto Nazionale dei Tumori di Milano, Milano, Italy

${ }^{8}$ Pediatric Surgery, Women's and Children's Health Department, University of Padua, Padua, Italy

${ }^{9}$ Pediatric Surgery Department, University of Palermo, Palermo, Italy

\section{Correspondence}

Maria Debora De Pasquale, Hematology/ Oncology and Stem Cell Transplantation Department, Bambino Gesù Children's Hospital, Piazza Sant'Onofrio, 4, 00165 Rome, Italy. Email:mdebora.depasquale@opbg.net

\begin{abstract}
Background: Malignant germ cell tumors (GCTs) are a heterogeneous group of rare neoplasms in children. Optimal outcome is achieved with multimodal therapies for patients with both localized and advanced disease, especially after the introduction of platinum-based chemotherapy regimens. In this respect, data on salvage treatment for children with relapsed or platinum-refractory disease are still limited.

Methods: Retrospective analysis of data regarding patients affected by malignant GCTs with platinum-refractory or relapsed disease after first-line treatment according to AIEOP TCGM 2004 protocol was conducted.

Results: Twenty-one patients, 15 females and 6 males, were considered for the analysis. All 21 patients received second-line conventional chemotherapy (SLCT), two of these immediately after surgery for local relapse removal. Two patients showed a progression of disease during SLCT and died of disease shortly thereafter, whereas 19 patients were in partial remission (PR) or complete remission (CR) after SLCT. Treatment after SLCT consisted in surgery on residual tumor mass (9/19) followed by high dose of chemotherapy (HDCT) with autologous hematopoietic stem cell support (16/19). The overall survival (OS) and event-free survival of the whole populations are $71 \%$ and $66.6 \%$, respectively. Platinum-refractory patients OS is $54.5 \%$ compared with $91.5 \%$ of the relapsed group. There were no treatment-related deaths.

Conclusion: SLCT followed or not by HDCT is an effective salvage treatment for children with relapsed/refractory GCTs. However, the role of HDCT following SLCT needs to be further investigated, especially regarding the identification of specific patient subgroups, which can benefit from this more intensive treatment.
\end{abstract}

KEYWORDS

germ cell tumors, pediatric tumors, high-dose chemotherapy, refractory tumors, relapsed tumors 


\section{INTRODUCTION}

Germ cell tumors (GCTs) are very rare in children, representing only $3 \%$ of all pediatric neoplasms.

In general, children diagnosed with GCTs and treated with cisplatinbased chemotherapy have an excellent prognosis, with an overall survival (OS) rate estimated at $\sim 80 \%$ to $85 \%$. However, $15 \%$ to $20 \%$ of these patients still die of their disease. ${ }^{1-9}$ Efforts to improve prognosis in this subgroup of patients have been made over the years, typically focusing on new chemotherapy regimens and the reduction of therapy side effects. Nonetheless, data and experience regarding secondline treatment for relapsed/refractory GCT in the pediatric setting are sparse, especially with regard to long-term outcome. ${ }^{10-12}$

We present the experience of the Associazione Italiana di Ematologia ed Oncologia Pediatrica (AIEOP) concerning treatment of children with relapsed/refractory malignant GCTs after first-line platinumbased chemotherapy.

\section{2 | METHODS}

Retrospective analysis was carried out of data regarding patients relapsed or platinum refractory after treatment according to the AIEOP TCGM 2004 protocol (for treatment of malignant GCTs). According to the World Health Organization/International Agency for Research on Cancer classification, malignant GCTs include seminoma, embryonal carcinoma, yolk sac tumor (YST), choriocarcinoma, and teratoma with somatic-type malignancies. ${ }^{13}$ The staging system used in this protocol for malignant tumors was as reported elsewhere. ${ }^{7-9}$ A centralized pathology review was possible, but not mandatory. Patients with malignant GCT, relapsed or platinum refractory, were included in this study. Platinum-refractory disease was defined, as in the adult setting, as disease relapse or progression (more than $25 \%$ increase in tumor size, new lesions, and/or rising tumor markers) in the four weeks following platinum-containing treatment. ${ }^{14}$ Data regarding sex, age, site and histopathological subtype of primary tumor, treatment applied, final status, type of relapse, treatment of relapse, and follow-up were collected and analyzed.

\section{1 | Response criteria}

Patients with normal tumor markers and no imaging abnormalities were classified as complete remission (CR). Patients with negative imaging and lower tumor markers, or with imaging abnormalities at the tumor site and declining/normalizing tumor markers, were classified as partial remission (PR). Patients with a more than $25 \%$ increase in tumor size, new lesions, and/or rising tumor markers were classified as progressive disease (PD).

\subsection{Patient survival analysis and statistical analysis}

The Kaplan-Meier method was used to estimate OS probabilities; differences between groups were compared with the log-rank test.
Hazard ratio for death was calculated with $95 \%$ confidence interval (Cl). Graph generation and statistical analyses were performed using Prism version 8.0 software (GraphPad).

\section{3 | RESULTS}

From 2004 to 2018, 252 patients with extracranial malignant GCTs were enrolled in the AIEOP TCGM 2004 protocol. The site of primary tumor was testis in 99 patients, ovary in 77 patients, mediastinum in 10 patients, sacrococcygeal in 45 patients, and extragonadal site other than mediastinum/sacrococcygeal in 21 patients.

Twenty-one patients, 15 females and 6 males, were considered for the analysis. Two of the 21 patients were considered for the analysis, but were not enrolled in the protocol: one patient due to rhabdomyosarcoma component in the initial specimen, and the second patient due to previous treatment with a different platinum-based chemotherapy. At diagnosis, they had a median age of 21 months (range, 1-214). The primary tumor site was sacrococcygeal in 11 patients, mediastinum in 1 patient, other extragonadal sites in 2 patients, ovary in 3 patients, and testis in 4 patients. Eleven patients presented metastasis at diagnosis. All patients had nonseminomatous GCT (Table 1).

All patients but one received four courses of PEB (cisplatin $25 \mathrm{mg} / \mathrm{m}^{2}$ days 1 to 4 , etoposide $100 \mathrm{mg} / \mathrm{m}^{2}$ days 1 to 4 , bleomycin $15 \mathrm{mg} / \mathrm{m}^{2}$ day 2) as first-line treatment.

Eleven patients (patients from numbers 1 to 11 ) resulted refractory to first-line cisplatin-based chemotherapy. The other ten patients relapsed at a median of six months after the end of first-line treatment (range, 1-13 months): relapses were local in six cases, and metastatic or with tumor marker increase only in the other four.

After relapse/progression, all 21 patients received second-line conventional chemotherapy (SLCT), two of these (patients 4 and 17) immediately after surgery consisting of removal of paravertebral relapse. The most commonly used regimen in 19 of 21 patients was based on carboplatin, ifosfamide, and etoposide (ICE: ifosfamide $1.8 \mathrm{~g} / \mathrm{m}^{2}$ days 1 to 5 , carboplatin $400 \mathrm{mg} / \mathrm{m}^{2}$ days 1 and 2, etoposide $100 \mathrm{mg} / \mathrm{m}^{2}$ days 1 to 5). Patients received a median of three courses of ICE (range, 24). Patient 13, with tumor marker increase after two courses of ICE, received two courses of GOP (gemcitabine $800 \mathrm{mg} / \mathrm{m}^{2}$ and paclitaxel $80 \mathrm{mg} / \mathrm{m}^{2}$ on days $1+8$, and oxaliplatin $130 \mathrm{mg} / \mathrm{m}^{2}$ on day 1 of a threeweek cycle) with reduction in tumor markers. Two patients (patients 3 and 5) showed a progression of disease after two and four courses of ICE and died of disease shortly thereafter. The other 19 patients were in PR or CR (patients 15 to 18) after SLCT.

Nine patients underwent surgery on residual tumor mass after SLCT (see Table 1).

After surgery, five more patients reached CR (patients 4, 10, 14, 16, and 17). Of the 19 patients in PR or CR after SLCT, 16 received highdose chemotherapy (HDCT) with autologous hematopoietic stem cell support. Conditioning regimen was based on thiotepa and cyclophosphamide in 15 of 16 patients. Patient 21 received two courses of HDCT (etoposide, thiotepa, and cyclophosphamide for the first course, 


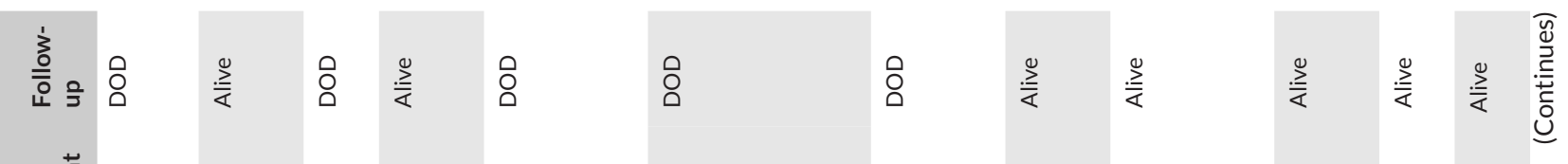

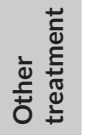

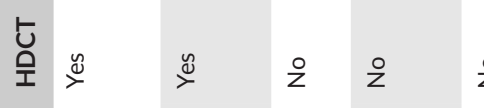

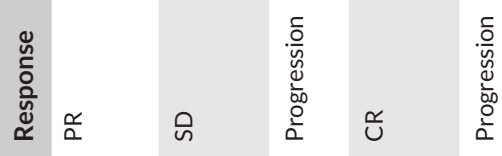

เ

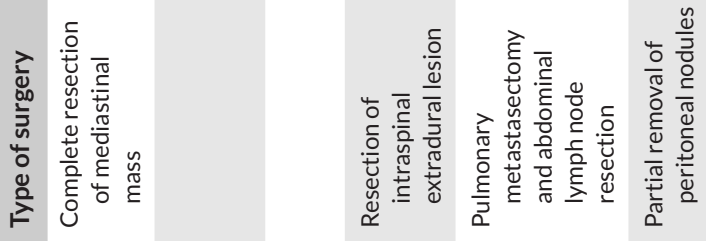

$\cong \quad \cong \quad \cong \quad \simeq \quad \cong \quad \simeq$

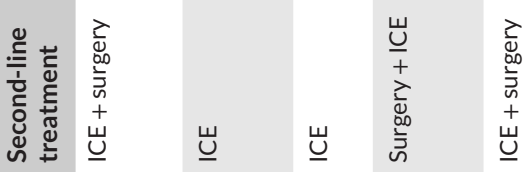

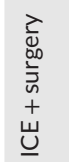

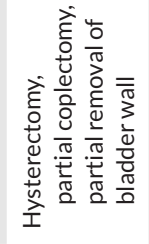

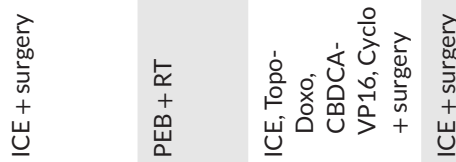

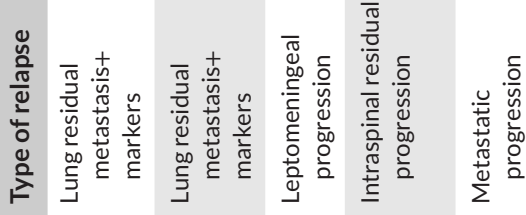

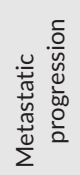

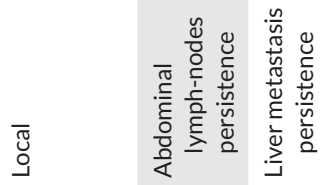

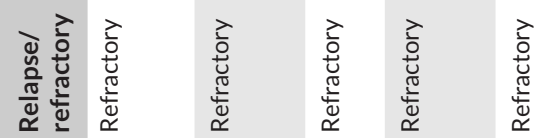

毫

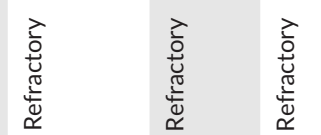

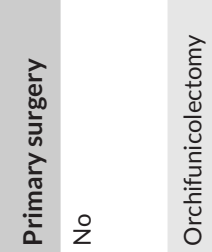
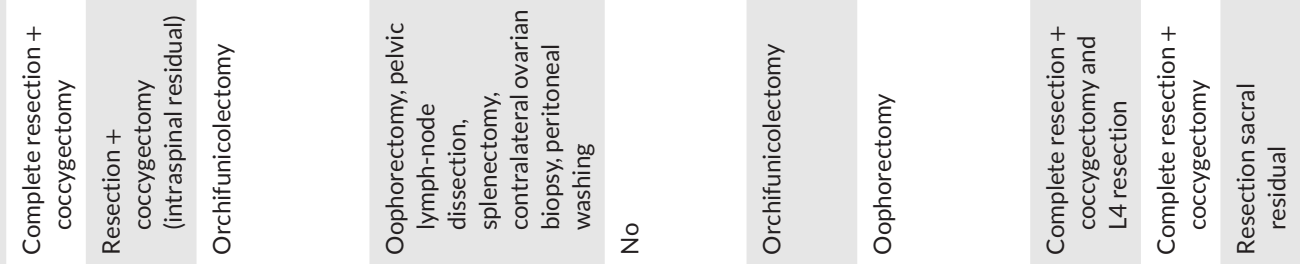

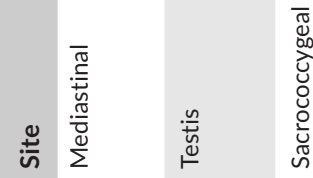

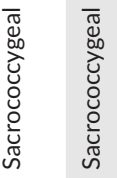

善

ํํำ

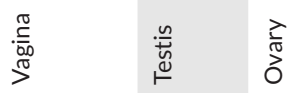

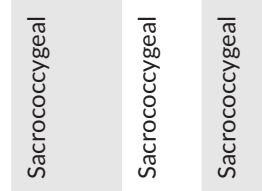

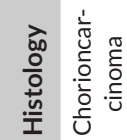

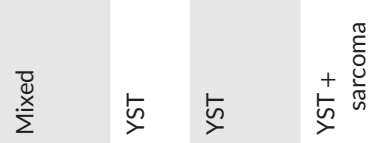

妾产豙

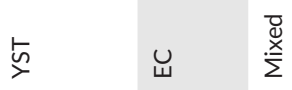

55

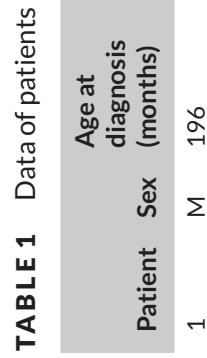




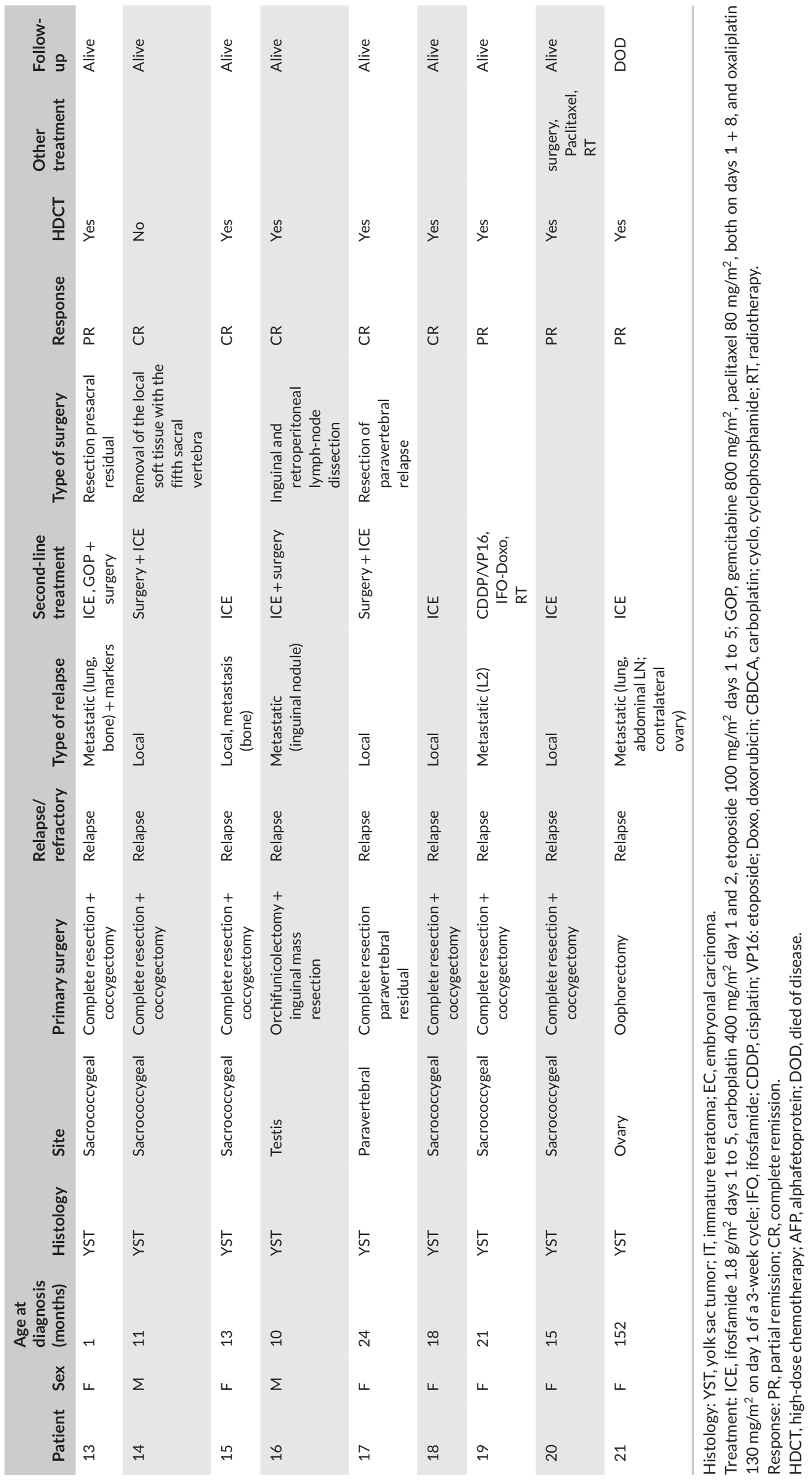



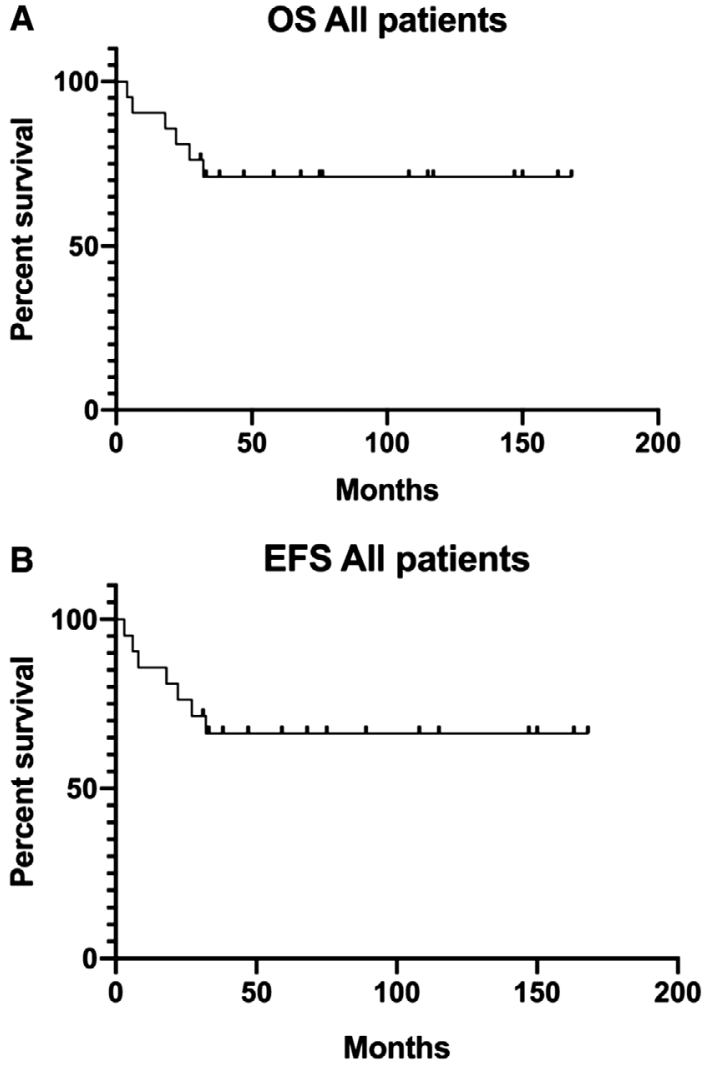

FIGURE 1 All patients: OS (A) and EFS (B)

thiotepa melphalan for the second course). Five patients did not receive $\mathrm{HDCT}$, in two cases because of disease progression and rapid death during SLCT (patients 3 and 5), in the other three cases because of physician decision for toxicity during SLCT (patient 6) and complete remission (CR) after ICE and surgery (patients 4 and 14).

One patient (patient 20) had a second local relapse after HDCT; he was treated with third-line chemotherapy based on taxane, surgery, and radiotherapy with maintenance of a third CR.

At follow-up, 15 of 21 patients are alive after second-line/thirdline treatment, whereas six patients died of disease at a median of 77 months from relapse (range, 19-161).

OS and event-free survival (EFS) of the entire cohort are $71 \%$ and $66.6 \%$, respectively (Figure 1). Platinum-refractory patients (patients 1 to 11 ) OS is $54.5 \%$ compared with $91.5 \%$ of the relapsed group (patients 12 to 21$)(P=0.0738)$ (Figure 2$)$.

Six patients presented episodes of bacteremia during SLCT. Two patients presented grade III mucositis during HDCT. No other grade 3 or higher nonhematologic toxic effects (Common Terminology Criteria for Adverse Events (CTCAE) v5.0, November 27, 2017) were recorded.

\section{4 | DISCUSSION}

Due to the rarity of the disease and the excellent outcome in the primary setting, consistent experiences on salvage treatment in children with relapsed /refractory GCTs are limited.

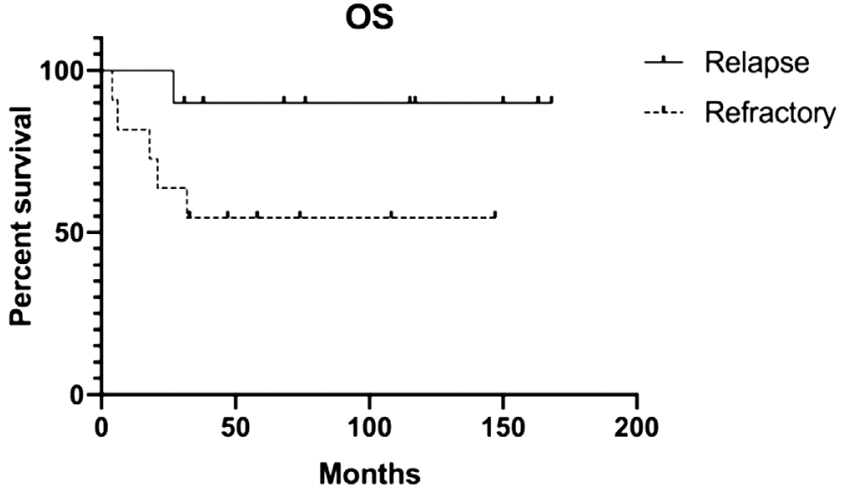

FIGURE 2 OS of relapsed and refractory patients

In 2016, Adra et al. published the largest single-institution series on HDCT in adults with relapsed GCTs, concluding that HDCT produced superior outcomes compared with standard-dose chemotherapy in all prognostic subgroups except the low-risk group. ${ }^{15}$ In this study, key prognostic factors in patients with relapsed GCT undergoing HDCT were: receiving HDCT in the third-line or subsequent setting, platinum-refractory disease, nonseminomatous histology, mediastinal primary tumor site, International Germ Cell Cancer Collaborative Group intermediate- or poor-risk disease at onset and hCG > = $1000 \mathrm{mIU} / \mathrm{mL}$ at the start of HDCT. ${ }^{15,16}$

The question about the superiority of HDCT versus conventionaldose chemotherapy is currently being analyzed in a multicentric American and European prospective randomized trial (TIGER trial, NCT02375204).

In our limited series, SLCT followed or not by surgery led to CR or PR in 18 of 21 patients. It is still unclear whether in these cases HDCT is necessary to maintain the obtained good response. In the pediatric setting, the role of HDCT in improving the outcome of patients with high-risk Ewing sarcoma and neuroblastoma is well established. However, HDCT in patients with high-risk soft-tissue sarcomas results in no significant improvement in outcome compared with standard-dose chemotherapy. ${ }^{17-20}$

De Giorgi et al. have investigated HDCT in children with relapsed extragonadal GCT. ${ }^{10}$ In this paper, 23 patients with extragonadal GCT, including GCT of the central nervous system, received HDCT as consolidation after SLCT. EFS was 43\%, lower than in our experience, but it should be taken into account that patients with gonadal site were not included in the study, while 9 patients with primitive central nervous system GCT and patients in second or third relapse were included. In different experiences concerning adults with GCTs, use of HDCT in second or third relapse seems to give a worse prognosis. ${ }^{15,21,22}$

Given the small size of our population, it is impossible to formulate a definitive judgment about the impact of HDCT on outcome. Only six patients in our series did not receive HDCT and three of these died of disease. However, two of them experienced a disease progression after SLCT and were thus not eligible for consolidation with HDCT. Two patients in CR after ICE and surgery (patients 4 to 14) did not undergo HDCT due to physician decision and are alive 
without disease. In our opinion, in the group of patients with local relapse, standard-dose chemotherapy, and surgery could be effective treatment.

Another interesting open question concerns which SLCT to use. There is no consensus about this issue in adult series as well. Pashankar et al. ${ }^{11}$ reported more than $50 \%$ of children in PR after two courses of TIP (paclitaxel $135 \mathrm{mg} / \mathrm{m}^{2}$ /day on day 1, ifosfamide $1800 \mathrm{mg} / \mathrm{m}^{2}$ on days 1 to 5 , and carboplatin with AUC 6.5 on day 1). In our experience, after SLCT based on "ICE," 19 of 21 patients reached PR or CR. Both of these points of discussion need further investigation in larger populations.

Focusing on the outcome, as reported by Adra et al. and similar to other studies in adults, ${ }^{15,23}$ patients with platinum-refractory disease seem to have worse prognosis compared with other groups, and potential future treatment options are under investigation for this subgroup of poor-prognosis patients. ${ }^{24-27}$ In our series, five of the six patients who died had refractory disease. The OS of this subgroup is only $54.5 \%$ compared with $91.5 \%$ of patients relapsed after firstline treatment, and the median OS was 33 months (95\% Cl, 4-147) (average 49.81 months) in the refractory patients versus 95.5 months (average 95.3 months) (95\% Cl, 27-168) in the relapse patients group ( $P=0.0738$; HR for death $5.637 ; 95 \% \mathrm{Cl}, 1.134-28.01$ ) (Figure 2). It can be speculated that patients with platinum-refractory disease need more intensive treatment in second-line setting compared with patients relapsed after first-line treatment.

Adult patients at relapse are stratified into five risk groups based on primary site and histology, response to prior treatment, progressionfree interval, tumor marker level, and the presence of liver, bone, and brain metastasis. ${ }^{28}$ It remains to be determined whether this stratification could also be used in the pediatric setting to guide the choice of treatment. The question of whether children with relapsed/refractory GCT could be cured using only SLCT combined with local treatment modalities, or whether HDCT consolidation is also necessary, is still open.

Contrary to other experiences, ${ }^{29}$ in our series, local control does not seem to impact upon survival during first-line treatment (all relapsed patients underwent complete removal of residual tumor at diagnosis) and at relapse. Faure-Conter et al. reported data on salvage treatment in 19 patients with relapsed and refractory GCTs; although outcome is worse than in our experience, EFS is significantly better in patients undergoing surgery compared with patients with unresectable tumors. ${ }^{30}$ Wessalowski et al. underlined the importance of local tumor control, achieved in this case with PEI-regional deep hyperthermia and tumor resection with or without RT. They recorded a fiveyear EFS of $62 \%$ and five-year EFS of $72 \%{ }^{31}$

In our experience, 4 of 11 patients (36\%) who underwent surgery at relapse died of disease compared with 2 of $10(20 \%)$ who did not receive surgery. Two patients in this last group received RT as local control and are alive (patients 8 and 19). The French study suggests considering the use of RT for unresectable tumors as well. ${ }^{30}$ Hence, even considering the small number of patients, it could be speculated that patients undergoing surgery may be those who have chemoresistant disease.
As previously reported, age (infants, children, and adolescents) and/or histologic subtypes ${ }^{32}$ have a significant influence on the outcome of malignant GCTs. ${ }^{33}$ In our limited series, age seems to have a prognostic value since all six patients older than 10 years had refractory disease. Moreover, five of these six patients died of disease, confirming the poor outcome of this population of patients.

In conclusion, SLCT followed or not by HDCT is an effective salvage treatment for children with relapsed/refractory GCTs. The role of HDCT after SLCT needs to be further investigated, in particular with regard to the identification of specific subgroups that could benefit from this more intensive treatment.

\section{CONFLICTS OF INTEREST}

We have no conflicts of interest to disclose.

\section{AUTHORS' CONTRIBUTIONS}

MDDP, PD, and MT contributed to conception, data collection and analysis, and writing of the manuscript; $\mathrm{AC}$ contributed to writing and literature review; RB, MC, GB, FS, AI, DB, PDA, and FS contributed to data collection and critical review of the manuscript; EM contributed to statistical analysis and critical review of the manuscript.

\section{DATA AVAILABILITY STATEMENT}

The data that support the findings of this study are available on request from the corresponding author. The data are not publicly available due to privacy or ethical restrictions.

\section{ORCID}

Maria Debora De Pasquale (D) https://orcid.org/0000-0003-1082-6810

Alessandro Crocoli (iD https://orcid.org/0000-0003-2157-4233

Gianni Bisogno (iD https://orcid.org/0000-0003-4462-5523

Filippo Spreafico (D) https://orcid.org/0000-0002-5587-3509

Alessandro Inserra (iD https://orcid.org/0000-0002-5663-8674

Patrizia Dall'Igna (iD https://orcid.org/0000-0002-3822-3272

Evelina Miele (iD https://orcid.org/0000-0002-4747-1032

Monica Terenziani (iD https://orcid.org/0000-0002-7080-6718

\section{REFERENCES}

1. Gobel U, Schneider DT, Calaminus G, Haas RJ, Schmidt P, Harms D. Germ-cell tumors in childhood and adolescence. GPOH MAKEl and the MAHO study groups. Ann Oncol. 2000;11(3):263-271.

2. Lo Curto M, Lumia F, Alaggio R, et al. Malignant germ cell tumors in childhood: results of the first Italian cooperative study "TCG 91". Med Pediatr Oncol. 2003;41(5):417-425.

3. Cushing B, Giller R, Cullen JW, et al. Randomized comparison of combination chemotherapy with etoposide, bleomycin, and either high-dose or standard-dose cisplatin in children and adolescents with high-risk malignant germ cell tumors: a pediatric intergroup study-Pediatric Oncology Group 9049 and Children's Cancer Group 8882. J Clin Oncol. 2004;22(13):2691-2700. 
4. Poynter JN, Amatruda JF, Ross JA. Trends in incidence and survival of pediatric and adolescent patients with germ cell tumors in the United States, 1975 to 2006. Cancer. 2010;116(20):4882-4891.

5. Gobel U, Calaminus G, Schneider DT, Schmidt P, Haas RJ. Management of germ cell tumors in children: approaches to cure. Onkologie. 2002;25(1):14-22.

6. Gobel U, Schneider DT, Calaminus G, et al. Multimodal treatment of malignant sacrococcygeal germ cell tumors: a prospective analysis of 66 patients of the German cooperative protocols MAKEI 83/86 and 89. J Clin Oncol. 2001;19(7):1943-1950.

7. De Pasquale MD, Crocoli A, Conte $M$, et al. Mediastinal germ cell tumors in pediatric patients: a report from the Italian Association of Pediatric Hematology and Oncology. Pediatr Blood Cancer. 2016;63(5):808-812.

8. Terenziani M, De Pasquale MD, Bisogno G, et al. Malignant testicular germ cell tumors in children and adolescents: the AIEOP (Associazione Italiana Ematologia Oncologia Pediatrica) protocol. Urol Oncol. 2018;36(11):502.e507-502.e513.

9. Terenziani M, Bisogno G, Boldrini R, et al. Malignant ovarian germ cell tumors in pediatric patients: the AIEOP (Associazione Italiana Ematologia Oncologia Pediatrica) study. Pediatr Blood Cancer. 2017;64(11). https://doi.org/10.1002/pbc.26568.

10. De Giorgi U, Rosti G, Slavin S, et al. Salvage high-dose chemotherapy for children with extragonadal germ-cell tumours. $\mathrm{Br} J$ Cancer. 2005;93(4):412-417.

11. Pashankar F, Frazier AL, Krailo M, et al. Treatment of refractory germ cell tumors in children with paclitaxel, ifosfamide, and carboplatin: a report from the Children's Oncology Group AGCT0521 study. Pediatr Blood Cancer. 2018;65(8):e27111.

12. Hou JY, Liu HC, Yeh TC, et al. Treatment results of extracranial malignant germ cell tumor with regimens of cisplatin, vinblastine, bleomycin or carboplatin, etoposide, and bleomycin with special emphasis on the sites of vagina and testis. Pediatr Neonatol. 2015;56(5):301-306.

13. Eble JN Sauter G, Epstein J. Pathology and Genetics of Tumours of the Urinary System and Male Genital Organs. Lyon: IARC Press; 2004.

14. Kollmannsberger C, Nichols C, Bokemeyer C. Recent advances in management of patients with platinum-refractory testicular germ cell tumors. Cancer. 2006;106(6):1217-1226.

15. Adra N, Abonour R, Althouse SK, Albany C, Hanna NH, Einhorn LH. High-dose chemotherapy and autologous peripheral-blood stem-cell transplantation for relapsed metastatic germ cell tumors: the Indiana University experience. J Clin Oncol. 2017;35(10):1096-1102.

16. International Germ Cell Consensus Classification: a prognostic factorbased staging system for metastatic germ cell cancers. Internationa Germ Cell Cancer Collaborative Group. J Clin Oncol. 1997;15(2):594603.

17. Valteau-Couanet D, Benhamou E, Vassal G, et al. Consolidation with a busulfan-containing regimen followed by stem cell transplantation in infants with poor prognosis stage 4 neuroblastoma. Bone Marrow Transplant. 2000;25(9):937-942.

18. Yalcin B, Kremer LC, van Dalen EC. High-dose chemotherapy and autologous haematopoietic stem cell rescue for children with high-risk neuroblastoma. Cochrane Database Syst Rev. 2015(10). Cd006301.

19. Drabko K, Raciborska A, Bilska K, et al. Consolidation of first-line therapy with busulphan and melphalan, and autologous stem cell rescue in children with Ewing's sarcoma. Bone Marrow Transplant. 2012;47(12):1530-1534.
20. Peinemann F, Enk H, Smith LA. Autologous hematopoietic stem cell transplantation following high-dose chemotherapy for nonrhabdomyosarcoma soft tissue sarcomas. Cochrane Database Syst Rev. 2017;4. Cd008216.

21. Oing C, Lorch A. The role of salvage high-dose chemotherapy in relapsed male germ cell tumors. Oncol Res Treat. 2018;41(6):365-369.

22. Zschabitz S, Distler FA, Krieger B, et al. Survival outcomes of patients with germ cell tumors treated with high-dose chemotherapy for refractory or relapsing disease. Oncotarget. 2018;9(32):2253722545.

23. Einhorn LH, Williams SD, Chamness A, Brames MJ, Perkins SM, Abonour R. High-dose chemotherapy and stem-cell rescue for metastatic germ-cell tumors. N Engl J Med. 2007;357(4):340-348.

24. Oing C, Seidel C, Bokemeyer C. Therapeutic approaches for refractory germ cell cancer. Expert Rev Anticancer Ther. 2018 18(4):389-397.

25. Adra N, Einhorn LH, Althouse SK, et al. Phase II trial of pembrolizumab in patients with platinum refractory germ-cell tumors: a Hoosier Cancer Research Network Study GU14-206. Ann Oncol. 2018 Jan 1;29(1):209-214

26. Necchi A, Lo Vullo S, Giannatempo P, et al. Pazopanib in advanced germ cell tumors after chemotherapy failure: results of the open-label, single-arm, phase 2 Pazotest trial. Ann Oncol. 2017 Jun 1;28(6):13461351.

27. Zschäbitz S, Lasitschka F, Hadaschik B, et al. Response to antiprogrammed cell death protein-1 antibodies in men treated for platinum refractory germ cell cancer relapsed after high-dose chemotherapy and stem cell transplantation. Eur J Cancer. 2017 May;76:1-7.

28. Lorch A, Beyer J, Bascoul-Mollevi C, et al. Prognostic factors in patients with metastatic germ cell tumors who experienced treatment failure with cisplatin-based first-line chemotherapy. J Clin Oncol. 2010;28(33):4906-4911.

29. Schneider DT, Wessalowski R, Calaminus G, et al. Treatment of recurrent malignant sacrococcygeal germ cell tumors: analysis of 22 patients registered in the German protocols MAKEI 83/86, 89, and 96. J Clin Oncol. 2001;19(7):1951-1960.

30. Faure-Conter C, Orbach D, Cropet C, et al. Salvage therapy for refractory or recurrent pediatric germ cell tumors: the French SFCE experience. Pediatr Blood Cancer. 2014;61(2):253-259.

31. Wessalowski R, Schneider DT, Mils O, et al. Regional deep hyperthermia for salvage treatment of children and adolescents with refractory or recurrent non-testicular malignant germ-cell tumours: an openlabel, non-randomised, single-institution, phase 2 study. Lancet Oncol. 2013;14(9):843-852.

32. Nogales FF, Jimenez RE. Pathology and Biology of Human Germ Cell Tumors. Berlin: Springer; 2019.

33. Oosterhuis JW, Looijenga LH. Testicular germ-cell tumours in a broader perspective. Nat Rev Cancer. 2005;5(3):210-222.

How to cite this article: De Pasquale MD, D'Angelo P, Crocoli A, et al. Salvage treatment for children with relapsed/refractory germ cell tumors: The Associazione Italiana Ematologia Oncologia Pediatrica (AIEOP) experience. Pediatr Blood Cancer. 2020;67:e28125. https://doi.org/10.1002/pbc.28125 